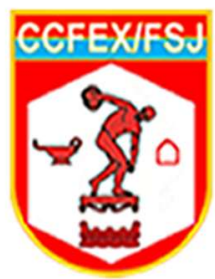

Revista de Educação Física

Joumal of Physical Education

Bome page: wnm revistadeeduoacaofisioa.com

Artigo Original

Original Article

\title{
Correlação e concordância do escore do Frequency - Intensity - Time Index of Kasari com o volume de oxigênio máximo
}

\section{Correlation and Agreement of FIT Score from Frequency - Intensity - Time Index of Kasari with the Maximum Oxygen Intake}

\author{
Vinícius Jacondino lahnke ${ }^{1}$; Aline Tito Barbosa Silva² MD; Angela Nogueira Neves ${ }^{\S 1}$ PhD
}

Recebido em: 30 de dezembro de 2019. Aceito em: 07 de maio de 2020.

Publicado online em: 18 de maio de 2020.

DOI: $10.37310 /$ ref.v88i4.862

\section{Resumo}

Introdução: O Frequency-Intensity-Time Index of Kasari avalia a prática de exercício físico em termos de intensidade, frequência e duração, podendo o produto destes parâmetros (FIT) ser como indicador de aptidão cardiorrespiratória.

Objetivo: Avaliar a correlação e concordância do FIT com volume de oxigênio máximo $\mathrm{VO}_{2 \max }$ em medidas direta e indireta.

Métodos: Estudo metodológico com seleção amostral do tipo não probabilística por conveniência, do qual participaram alunos do curso de Instrutores de Educação Física (CI) e membros da família militar $(\mathrm{n}=59)$. A amostra foi dividida em dois grupos de estimação do $\mathrm{VO}_{2 \text { max: }}$ protocolo de rampa, utilizando expirômetro ( $\left.\mathrm{VO}_{2 \text { maxrampa }}\right)$, aplicado na família militar; e teste de Cooper $\left(\mathrm{VO}_{2 \operatorname{maxcooper}}\right)$, aplicado nos alunos. Todos responderam a mesma versão do FIT. A correlação foi estimada pelo do coeficiente de correlação de Pearson e a concordância foi avaliada por meio do coeficiente de concordância $(\rho c)$ e da análise gráfica de Bland e Altman. Os softwares SPSS 15 e MedCalc foram usados.

Resultados: A amostra foi composta por 24 alunos do CI $(26,5 \pm$ 2,39 anos) e 35 membros da família militar (45,28 \pm 10,79 anos). Houve correlação significante e fraca apenas entre FIT e $\mathrm{VO}_{2 \text { maxcooper. }}$ Os coeficientes de concordância do FIT foram pobres e desprezíveis

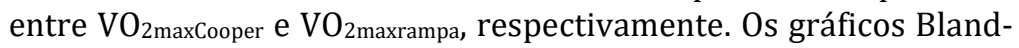
Altman exibiram viés de diferença considerável, o que indica margens amplas de sub e superestimação do $\mathrm{VO}_{2 \text { max. }}$.

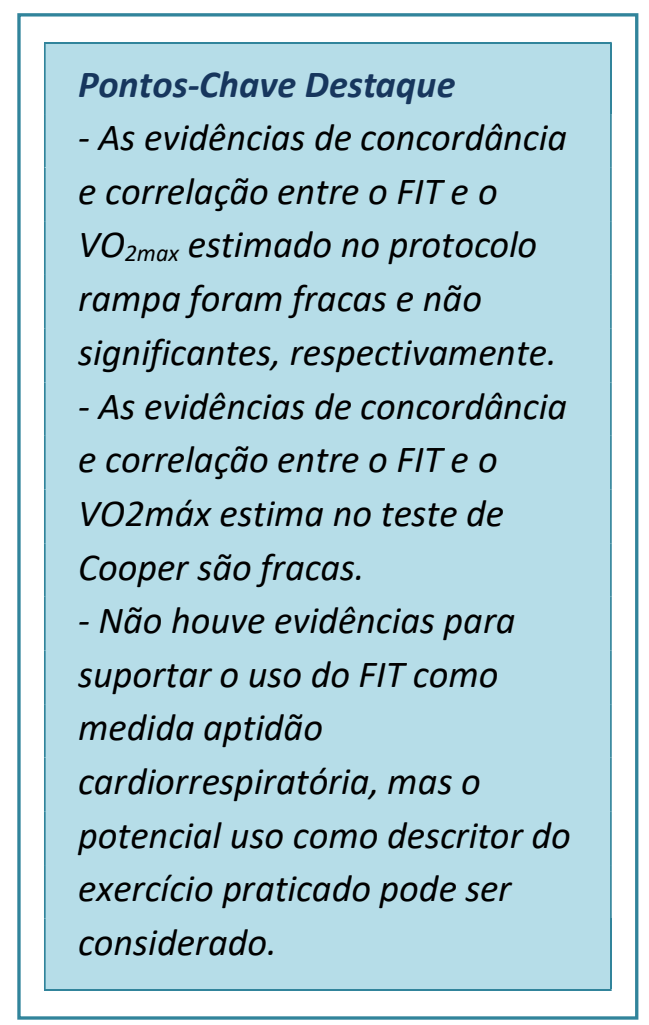

Conclusão: 0 Frequency-Intensity-Time Index of Kasari parece ser adequado para descrever de exercício físico praticado, mas o uso do FIT precisa ser melhor investigado.

Palavras-chave: aptidão física, aptidão cardiorrespiratória, autorrelato.

\begin{abstract}
Introduction: The Frequency-Intensity-Time Index of Kasari evaluates physical activity practice in terms of frequency, intensity, and length, being the product of these parameters (FIT) interpret as an indicator of aerobic fitness.

Objective: To evaluate the correlation and agreement of (FIT) with maximum oxygen intake $\left(\mathrm{VO}_{2 \text { max }}\right)$ in direct
\end{abstract}

$\S$ Autor correspondente: Angela Nogueira Neves - e-mail: angelaneves.esefex@gmail.com

Afiliações: 'Escola de Educação Física do Exército (EsEFEx); ${ }^{2}$ Instituto de Pesquisa da Capacitação Física do Exército (IPCFEx). 
and indirect measures.

Methods: Methodological study, with non-probabilistic sampling, by convenience, in which students from the Physical Education Instructors (CI) course and members of the military family participated $(\mathrm{n}=59)$. The sample was divided into two $\mathrm{VO}_{2 \max }$ estimation groups: ramp protocol, using an expirometer ( $\mathrm{VO}_{2 \mathrm{max}}$ ramp), applied to the military family; and Cooper's test ( $\mathrm{VO}_{2 \text { maxCooper }}$, applied to students. They all answered the same version of FIT. The correlation was estimated using the Pearson correlation coefficient and the agreement was assessed using the agreement coefficient ( $\rho c$ ) and the graphic analysis by Bland and Altman. The SPSS 15 and MedCalc software were used.

Results: The sample was composed by 24 students from CI $(26,5 \pm$ 2,39 years old) and 35 members of military family $(45,28 \pm 10,79$ years old). A weak and significant correlation between FIT and $\mathrm{VO}_{2 \text { maxCooper }}$ was found. The agreement coefficient was week and despicable among FIT and $\mathrm{VO}_{2 \text { maxcooper }}$ and $\mathrm{VO}_{2 \text { maxRamp, respectively. }}$ The Bland-Altman graphs showed a large difference bias, which constitutes wide margins of underestimation and overestimation of $\mathrm{VO}_{2 m a x}$.

Conclusion: The Frequency-Intensity-Time Index of Kasari seems to be adequate to describe physical exercise, but the FIT use requires further investigation.

\section{Keypoints}

- Agreement and correlation between FIT and $\mathrm{VO}_{2 \max }$ estimated in the ramp protocol was weak and not significant, respectively.

- The evidence of agreement and correlation between the FIT and the $\mathrm{VO}_{2 \max }$ estimated in the Cooper test is weak.

- There was no evidence to support the use of FIT as a cardiorespiratory fitness measure, but the potential use as a descriptor of the exercise practiced can be considered.

\section{Correlação e concordância do escore do Frequency - Intensity - Time Index of Kasari com o volume de oxigênio máximo}

\section{Introdução}

A capacidade cardiorrespiratória é um componente da aptidão física, e está associada à realização de exercícios de alta intensidade muscular, dinâmicos, de intensidade moderada a vigorosa por períodos prolongados(1). O volume máximo de oxigênio consumido por unidade de tempo, representado por $\mathrm{VO}_{2 \max }$, é um parâmetro utilizado para mensuração e classificação da capacidade cardiorrespiratória. Essa variável é expressa em razão do consumo de oxigênio em mililitros por quilo de peso corporal a cada minuto (ml/kg.min-1) estando associada à capacidade funcional e à integração dos sistemas do corpo humano com influência no fornecimento, transporte, distribuição e utilização do oxigênio(1-3).
Os testes que estimam o $\mathrm{VO}_{2 \max }$ de forma indireta $o$ fazem por meio de equações baseadas no resultado de testes físicos submáximos e máximos(4). Estes testes aferem, por meio de cálculos da taxa metabólica a quantidade de oxigênio necessária para a realização de movimentos do corpo como o caminhar, o movimento de pedalar e a corrida; dentre os fatores que influenciam no resultado estão o tempo e a distância percorrida, frequência cardíaca, idade, sexo, nível de treinamento físico dentre outros(5). São mais simples, de baixo custo, podem ser aplicados a um maior número de indivíduos e possuem correlação moderadamente alta com o consumo máximo de oxigênio dos indivíduos(5). 
Manter o acompanhamento da aptidão física do militar, incluindo sua capacidade cardiorrespiratória é essencial para a avaliação da capacidade operativa, além de sua saúde. Ressalta-se que nos exércitos modernos, pretende-se a formação de um soldado de qualidade, que desenvolva seu trabalho em cenários muito diversificados e, às vezes, em condições extremas, o que exige grande versatilidade além da aptidão física(6). $O$ Exército Brasileiro observa, em seu manual de treinamento físico, que o militar deve estar, constantemente, preparado para suportar diferentes agentes estressores que podem surgir durante o combate, sejam eles físicos, psicológicos, ambientais e/ou nutricionais. Nesse contexto, ressalta-se a importância do treinamento físico e dos benefícios para a saúde proporcionados pela adaptação ao treinamento, e da maior eficiência no desempenho profissional(6).

A efetividade do treinamento físico pode ser inferida pelas avaliações quadrimestrais realizadas no Teste de Aptidão Física do Exército Brasileiro (TAF)(6). O TAF é constituído por parâmetros de avaliação neuromuscular (flexão de braços, abdominal supra, flexão na barra) e por teste indireto de avaliação da capacidade aeróbia, o teste de Cooper de 12 minutos(6,7). Importante salientar que os familiares do militar são atendidos pela rede conveniada e própria do Exército, sendo mais comumente usados nos ambientes clínicos, protocolos indiretos em ergômetros para estimação da aptidão cardiorrespiratória(8).

As características extremas de trabalho podem inviabilizar a execução do treinamento visando a manutenção da aptidão física, bem como a avaliação da capacidade física e cardiorrespiratória dos militares que estão desenvolvendo as atividades(6). Da mesma forma, quando serve em lugares afastados e de pouca estrutura - como nos Pelotões Especiais de Fronteira - a família do militar também pode ficar carente de estrutura de prática de exercício físico e acompanhamento clínico. Tendo isso em vista, outras formas de avaliação e acompanhamento, além dos testes supracitados, devem ser investigadas. Instrumentos do tipo autopreenchíveis, com comprovada correlação e concordância com os resultados de um teste direto, podem ser uma boa solução, pois geralmente são de baixo custo e fáceis de serem aplicados(9).

O Frequency-Intensity-Time Index of Kasari é uma escala autopreenchível, breve (com apenas três perguntas) desenvolvida na década de 1970(10). O instrumento avalia especificamente o comportamento de prática de exercício físico com o registro da intensidade, frequência e duração dos exercícios praticados(10). Pode-se gerar um escore a partir do produto da intensidade, frequência e duração do exercício, chamado de FIT (Frequency $\mathrm{x}$ Intesity $\mathrm{x}$ Time $=$ FIT score). Esse escore pode ser interpretado de duas formas: como um indicador do nível de atividade física ou como indicador no nível e condicionamento físico aeróbico(11). Esta última interpretação do FIT foi escolhida para ser investigada nessa pesquisa, pois se a mesma for pertinente, poderia ser usada em situações extremas, na qual o oficial de treinamento físico militar necessita de parâmetros para a prescrição de exercício, sem poder realmente conduzir um teste direto ou indireto da capacidade aerobia(12,13).

Neste sentido, visualizou-se a relevância de verificar-se a compatibilidade entre o indicador de condicionamento físico avaliado através das informações auto reportadas da intensidade, duração e tempo de exercício aeróbio normalmente realizado, com os testes de campo de Cooper e Ergômetro usando protocolo em rampa, cuja confiabilidade dos resultados que expressam o $\mathrm{VO}_{2 \max }$ estimado é conhecida cientificamente(5). A revisão de literatura indicou que o FIT obteve correlação com o $\mathrm{VO}_{2 \max }(10)$, mas, até onde se pode verificar, não houve nenhuma análise de concordância. De igual forma, a revisão de literatura indicou que nenhum estudo sobre a adequacidade do FIT, tanto como medida de nível de condicionamento aeróbico como medida de nível de prática de exercício físico havia sido já realizado em amostras de referências brasileiras.

O presente estudou visou verificar correlação e concordância entre o produto da intensidade, frequência e duração avaliados pelo Frequency-Intensity-Time Index of Kasari, o FIT, e o nível de capacidade cardiorrespiratória - através do $\mathrm{VO}_{2 \max }$ 
estimado, para verificar a pertinência da interpretação do FIT como estimativa de condicionamento físico. Em caso positivo, o FIT surgiria como uma alternativa confiável para determinação do nível de condicionamento aeróbio na impossibilidade da condução de testes em campo ou laboratório, devido à baixa disponibilidade de meios.

\section{Métodos}

\section{Desenho de estudo e amostra}

Estudo metodológico, com seleção amostral do tipo não probabilística, por conveniência. A população alvo foram membros da família militar e alunos oficiais do curso de Instrutor de Educação Física da Escola de Educação Física do Exército. O cálculo do tamanho amostral foi realizado via software G*Power(15); com os parâmetros: $\alpha=0,05,1$ $\beta=0,95$. O tamanho amostral mínimo recomendado foi de 32 participantes. Os critérios de inclusão foram ser praticante de exercícios físicos e contar com idade mínima de 18 anos. O critério de exclusão adotado foi a impossibilidade de realizar o teste para estimação do $\mathrm{VO}_{2 \max }$, por consequência de lesões, doenças ou impeditivos clínicos.

A amostra foi dividida em dois grupos de estimação do $\mathrm{VO}_{2 \max }$ : protocolo de rampa, utilizando expirômetro ( $\mathrm{VO}_{2 \text { maxrampa }}$ ), aplicado na família militar; e teste de Cooper $\left(\mathrm{VO}_{2 \mathrm{max} C o o p e r}\right)$, aplicado nos alunos.

\section{Aspectos éticos}

O presente estudo foi aprovado no Comitê de Ética em Pesquisa envolvendo seres humanos do CCFEx. C.A.A.E: 15425019.1.0000.9433. Todos os participantes assinaram o Termo de Consentimento Livre e Esclarecido (TCLE).

\section{Variáveis de estudo}

As variáveis desfecho estudadas foram o FIT e o $\mathrm{VO}_{2 \max }$ (medidas direta e indireta).

\section{Frequency-Intensity-Time Index of Kasari}

O Frequency-Intensity-Time Index of Kasari é uma avaliação de prática de atividade e de aptidão física. Trata-se de uma escala autopreenchível, que orienta o respondente a marcar a respostas que melhor descreve sua prática de exercício físico atual. É composta por apenas três perguntas, que avalia a prática de exercício físico considerando a intensidade, frequência e duração dos exercícios praticados(10) e, a partir do produto desses parâmetros, gera um escore (Frequency $\mathrm{x}$ Intesity $\mathrm{x}$ Time $=$ FIT score)(17). Sendo escala do tipo Likert, cada parâmetro é pontuado da seguinte forma: 1) Frequência: $1=<$ uma vez ao mês; $5=>$ seis vezes por semana; 2) Intensidade $(1=$ exercício aeróbico leve, $5=$ exercícios de alta intensidade; e 3) Duração (1 $=<\operatorname{dez}$ minutos por sessão, $4=>$ trinta minutos por sessão(10). A interpretação do FIT independe do sexo e idade do respondente e podem ser as seguintes: 1) Como indicador de nível de prática de exercício físico, sendo FIT $=100$ indicador muita atividade física e estilo de vida ativo; 80-99 ativo e saudável; 60-79 ativo; de 40 a 59 aceitável; 20-39 insuficiente prática de exercícios e $<20$ indicador de sedentarismo; e 2) Como indicador de aptidão cardiorrespiratória, sendo FIT $=100$ indicador de alto condicionamento; 80-99 muito bom; 60-79 bom; de 40 a 59 fraco; 20-39 pobre e $<$ 20 indicador de condicionamento aeróbico muito pobre(11). É esta última interpretação do FIT tomada para esta pesquisa.

\section{Volume máximo de oxigênio consumido por unidade de tempo $\left(\mathrm{VO}_{2 \max }\right)$}

$\mathrm{O}$ volume máximo de oxigênio consumido por unidade de tempo $\left(\mathrm{VO}_{2 \max }\right)$ foi estimado em método direto pelo protocolo de rampa $\left(\mathrm{VO}_{2 \text { maxrampa }}\right)(16)$ e em método indireto, pelo teste de Cooper: $\mathrm{VO}_{2 \max \text { Cooper }}$ (5).

\section{Protocolo de rampa ( $\mathrm{VO}_{2 \text { maxrampa) }}$}

Os participantes foram submetidos a um teste de esforço cardiopulmonar, aplicando-se um protocolo de rampa $\left(\mathrm{VO}_{2 \text { maxrampa }}\right)$, com a utilização de expirômetro, com o exercício realizado em ergômetro, do tipo esteira rolante (Imbramedmaster fabricada no Brasil), controlada pelo sistema ErgoMET, que comanda a velocidade e inclinação da esteira de acordo com estimativa do volume máximo de oxigênio para a idade e sexo dos avaliados.

Os parâmetros do teste foram individualizados e ajustados da seguinte forma: o volume máximo de oxigênio previsto para o sexo e idade, o tempo proposto de esforço, a velocidade inicial e final do teste, a inclinação inicial e final, e o tipo de exercício (corrida ou caminhada). 
A velocidade inicial do teste foi de $3 \mathrm{Km} / \mathrm{h}$ e a final máxima foi de $12 \mathrm{~km} / \mathrm{h}$, podendo o teste ser interrompido a uma velocidade menor por fadiga muscular ou solicitação do participante. A inclinação inicial foi de 0 graus e a final atingiu até 5 graus no máximo. Os incrementos de velocidade e inclinação ocorreram a cada 20 segundos partindo dos marcos de 0 graus e 3 $\mathrm{km} / \mathrm{h}$. As características da rampa foram escolhidas a pós avaliação clínica do participante, conduzida por medido cardiologista, para proporcionar uma duração do teste entre 8 a 12 minutos, seguindo a recomendação da American Heart Association(16). A fase de recuperação foi realizada com uma velocidade de $40 \%$ da máxima atingida por 3 minutos, para observação do comportamento cardiorrespiratório no retorno ao repouso.

O teste teve característica sintoma-limitante, sendo interrompido por solicitação do avaliado ou no aparecimento de algum critério de interrupção, de acordo com o American College of Sports and Medicine(1) com atenção aos seguintes sintomas: início de angina, tonturas, confusão, palidez, ausência do aumento da frequência cardíaca com uma maior intensidade do exercício, manifestações físicas ou verbais de fadiga extrema. Os participantes do estudo foram estimulados verbalmente durante a execução do teste. Os testes foram conduzidos no Laboratório de Fisiologia do Instituto de Pesquisa da Capacitação Física do Exército (IPCFEx).

\section{Teste de Cooper ( $\mathrm{VO}_{2}$ maxcooper)}

Para estimar indiretamente a aptidão cardiorrespiratória, utilizou-se o teste Cooper de 12 minutos $\left(\mathrm{VO}_{2 \text { maxCooper }}\right)(5)$. Os participantes foram submetidos ao protocolo na pista de atletismo da Escola de Educação Física do Exército (EsEFEx). A largada foi realizada com os voluntários alinhados na pista, e desenvolvida com raias livres. O balizamento da pista foi realizado a cada 50 metros com cones de identificação da distância percorrida. Houve um aquecimento livre de 10 minutos, após este período os participantes foram posicionados no dispositivo de largada para o início do procedimento. Ao comando de "às suas marcas" seguido de silvo de apito curto, iniciaram a corrida. A partir deste momento, correram sem interrupções durante
12 minutos a máxima distância possível, sendo registrada em metros ao final e utilizada apara estimarmos o $\mathrm{VO}_{2 \max }$ através da seguinte equação matemática(5):

$$
\left[\mathrm{VO}_{2 \max }(\mathrm{ml} / \mathrm{kg} \cdot \mathrm{min}-1)=(\text { metro-504,9/44,73)] }\right.
$$

\section{Procedimentos de coleta de dados}

Para coleta de dados da pesquisa, foram convidados os militares e membros da família militar inscritos para testes ergométricos no IPCFEx e os alunos do curso de instrutores da EsEFEx a participar. Oralmente, esclareceu-se acerca da coleta a ser realizada e os participantes foram convidados a participar, de forma voluntária, da pesquisa. Aos que aceitaram, apresentamos o TCLE para leitura e assinatura.

Para os voluntários da família militar, após esses procedimentos, foi entregue o Frequency-Intensity-Time Index of Kasari no qual o participante assinalou as respostas mais adequadas acerca da intensidade, duração e frequência do exercício físico por ele praticado.

Para os voluntários do Curso de Instrutores, pela dificuldade de garantir um espaço reservado em campo, o Frequency-IntensityTime Index of Kasari foi respondido no dia anterior à execução do teste Cooper de 12 minutos. O mesmo seguiu o padrão do teste de aptidão física do Exército Brasileiro, conforme descrito anteriormente

Como forma de retorno aos participantes, ao final da pesquisa, foi entregue o resultado da pesquisa e o valor $\mathrm{doVO}_{2 \max }$ estimado $\left(\mathrm{VO}_{2 \mathrm{maxCooper}}\right)$ através de e-mail, para acompanhamento do nível de condicionamento físico.

\section{Análise Estatística}

A análise da descrição dos dados obtidos foi realizada através de medidas de tendência central e dispersão. Para comparação dos resultados, utilizou-se o teste D'AgostinoPearson para verificação da normalidade das variáveis.

Procedeu-se com a análise de correlação de Pearson para os pares testados (FIT $x$ $\mathrm{VO}_{2 \text { maxCooper; }}$ FIT x $\mathrm{VO}_{2 \text { maxrampa), adotando-se }}$ como ínfimos valores menores ou iguais a 0,39 ; moderados valores entre 0,40 e 0,59 ; fortes os valores entre 0,90 a 0,60 e muito forte 
a correlação acima de 0,90. , apenas as correlações forte e muito fortes. Para a determinação de qual fração da variabilidade do teste $\mathrm{VO}_{2 \max }$ estimado é explicado pelo FIT, foram calculados os coeficientes de determinação (r2), através do quadrado do coeficiente de correlação de Pearson. Valores acima de $70 \%$ foram considerados mais adequados(18).

A concordância dos valores obtidos entre cada par de variáveis (FIT x VO $\mathrm{VO}_{2 \text { maxCooper; }}$ FIT

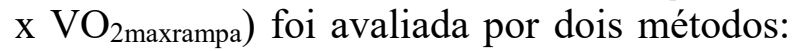
coeficiente de concordância (21) e análise gráfica de Bland e Altmann(22). Primeiro, pelo coeficiente de concordância $(\rho c=\rho \times \mathrm{Cb})$, onde: $\rho$ é o coeficiente de correlação de Pearson, que mede o quanto cada observação se desvia da linha de melhor ajuste, sendo uma medida de precisão; $\mathrm{Cb}$ é um fator de correção de viés que mede a distância que a linha de melhor ajuste se desvia da linha de $45^{\circ}$ até a origem sendo uma medida de acurácia $(19,20)$. Para o julgamento da qualidade da concordância, foram adotados os seguintes parâmetros: $\leq 0,90$ concordância pobre; entre 0,91 - 0,95 concordância moderada; entre 0,96 - 0,98 concordância substancial; $\geq 0,99$ concordância quase perfeita(21).

O segundo método adotado para avaliação da concordância foi a análise gráfica de Bland e $\operatorname{Altman}(22)$. Foi utilizado um limite de concordância (LC) de $95 \%$ pela seguinte equação: $\mathrm{LC}=(1.96 \times \mathrm{DP}) \pm$ Mdif; onde $\mathrm{DP}=$ desvio padrão e Mdif = média das diferenças. Em todas estas análises, foi adotado um nível de significância de $p<0,05$. O software SPSS 15 e o software MedCalc (versão 19.0.7) foram usados nas análises.

\section{Resultados}

A amostra do estudo foi composta por um total de 59 participantes, sendo 24 alunos do Curso de Instrutores, com idade média de 26,5 anos $(\mathrm{DP}=2,39$; máx $=34$; mín $=24)$, e 35 membros da família militar com média de idade de 45,28 anos ( $\mathrm{DP}=10,79$; máx $=77$; mín =19). As medidas de tendência central e valores de dispersão, assim como a significância do teste de normalidade dos dados pode ser visto na Tabela 1 . Na Tabela 2 , descrevem-se as categorias de aptidão cardiorrespiratória, conforme preconizado pelo FIT.

O teste de correlação de Pearson indicou não haver qualquer correlação estatisticamente significante entre o FIT e o $\mathrm{VO}_{2 \text { maxrampa }}(\mathrm{r}=$ $0,11, \mathrm{p}=0,55)$. Ademais, a parcela de variabilidade explicada do $\mathrm{VO}_{2 \text { maxrampa }}$ pelo FIT foi penas $1 \%(\mathrm{r} 2=0,01)$. Houve correlação positiva e moderada do FIT com $\mathrm{VO}_{2 \text { maxCooper }}$ $(\mathrm{r}=0,43 ; \mathrm{p}=0,02)$. Todavia, para os propósitos de presente estudo, esta correlação é fraca para ser considerada como relevante. A variância explicada do $\mathrm{VO}_{2 \text { maxcooper }}$ pelo FIT na amostra foi de $\mathrm{r} 2=0,18$ (Figura1).

Quanto à concordância, o coeficiente de concordância para o par de medidas FIT x $\mathrm{VO}_{2 \text { maxrampa }}$ foi pobre; $\rho \mathrm{c}=0,04$; sendo $\mathrm{Cb}=$ 0,41 . O coeficiente de concordância foi igualmente pobre para o FIT x $\mathrm{VO}_{2 \text { maxCooper, }}$ $\rho c=0,13$; sendo $C b=0,31$.

Mesmo com estes valores inferiores, procedeu-se com a análise gráfica de BlandAltman (Figura 2).

Para o par de medidas FIT x $\mathrm{VO}_{2 \text { maxrampa, a }}$ média das diferenças (viés) entre o FIT e o VO2máx obtido no protocolo rampa foi de 14,99 unidades, o que indica que, na média, o $\mathrm{VO}_{2 \max }$ mede 14,99 a mais que o FIT. Foi calculado o intervalo de confiança de $95 \%$, que varia entre $-25,74$ a 55,71. Estes resultados indicam que o FIT pode estar 55 unidades acima ou 25 unidades abaixo do $\mathrm{VO}_{2 \text { maxrampa }}$ na amostra. Ainda, a análise gráfica de BlandAltman, apontou que $5,71 \%$ dos dados estão fora da margem indicativa de concordância. $\mathrm{O}$ teste $t$ de Student de uma amostra (diferença entre as medidas) apontou que a concordância entre as duas medidas não se sustenta $(\mathrm{p}<0,05)$.

Já para o par de variáveis FIT x $\mathrm{VO}_{2 \text { maxCooper, }}$ foi possível verificar que o viés da diferença entre as medidas é de 7,8 unidades aproximadamente. Assim, o $\mathrm{VO}_{2 \max }$ mede 7,77 unidades a mais que o FIT, na média. $\mathrm{O}$ valor máximo de concordância foi calculado em 51,32 unidades e o valor mínimo em -35,77 unidades. Por fim, o teste $t$ de Student aponta haver concordância entre as medidas $(\mathrm{p}>0,05)$ (Tabela 3). 
Tabela 1 - Medidas de tendência central e dispersão dos dados do $\mathrm{VO}_{2 \max }$

\begin{tabular}{lcccc}
\hline Medidas & $\begin{array}{c}\text { FIT Família } \\
\text { militar } \\
(\mathbf{n = 3 5 )}\end{array}$ & $\begin{array}{c}\text { VO }_{\text {2max: }} \\
\text { rampa }\end{array}$ & $\begin{array}{c}\text { FIT } \\
\text { alunos CI } \\
(\mathbf{n = 2 4 )}\end{array}$ & $\begin{array}{c}\text { VO2max: } \\
\text { Cooper }\end{array}$ \\
\hline \hline $\mathrm{n}$ & 35 & 35 & 24 & 24 \\
Média & 56,03 & 41,04 & 60,83 & 57,06 \\
Mediana & 60,00 & 41,60 & 64,00 & 58,50 \\
Desvio Padrão & 20,30 & 7,052 & 23,65 & 3,71 \\
Mínimo & 10,00 & 24,90 & 27,00 & 49,02 \\
Máximo & 100,00 & 63,30 & 100 & 61,29 \\
\% 25-75 & $48,00-72,25$ & $36,02-45,75$ & $45,50-80,00$ & $53,48-60,18$ \\
$\boldsymbol{P}$ & 0,27 & 0,45 & 0,31 & 0,17 \\
\hline
\end{tabular}

$\mathbf{n}=$ tamanho amostral; Min = valor mínimo; Máx = valor máximo; \% = percentis; $\mathbf{C A}=$ condicionamento aeróbico;

$\boldsymbol{P}$ : p-valor resultado do teste de normalidade teste D'Agostino-Pearson.

Tabela 2 - Categorias de condicionamento aeróbico avaliado pelo FIT

\begin{tabular}{lcc}
\hline \multicolumn{1}{c}{ Categorias } & FIT Família Militar & FIT Alunos do CI \\
\hline \hline \% CA alto* & $2,88 \%$ & $20,83 \%$ \\
\% CA muito bom* & $20,00 \%$ & $12,50 \%$ \\
\% CA bom* & $28,57 \%$ & $33,34 \%$ \\
\% CA fraco* & $28,57 \%$ & $12,50 \%$ \\
\% CA pobre* & $17,14 \%$ & $20,83 \%$ \\
\% CA muito pobre* & $2,88 \%$ & $0 \%$ \\
\hline
\end{tabular}

*Classificado de acordo com as faixas de interpretação do FIT(11)
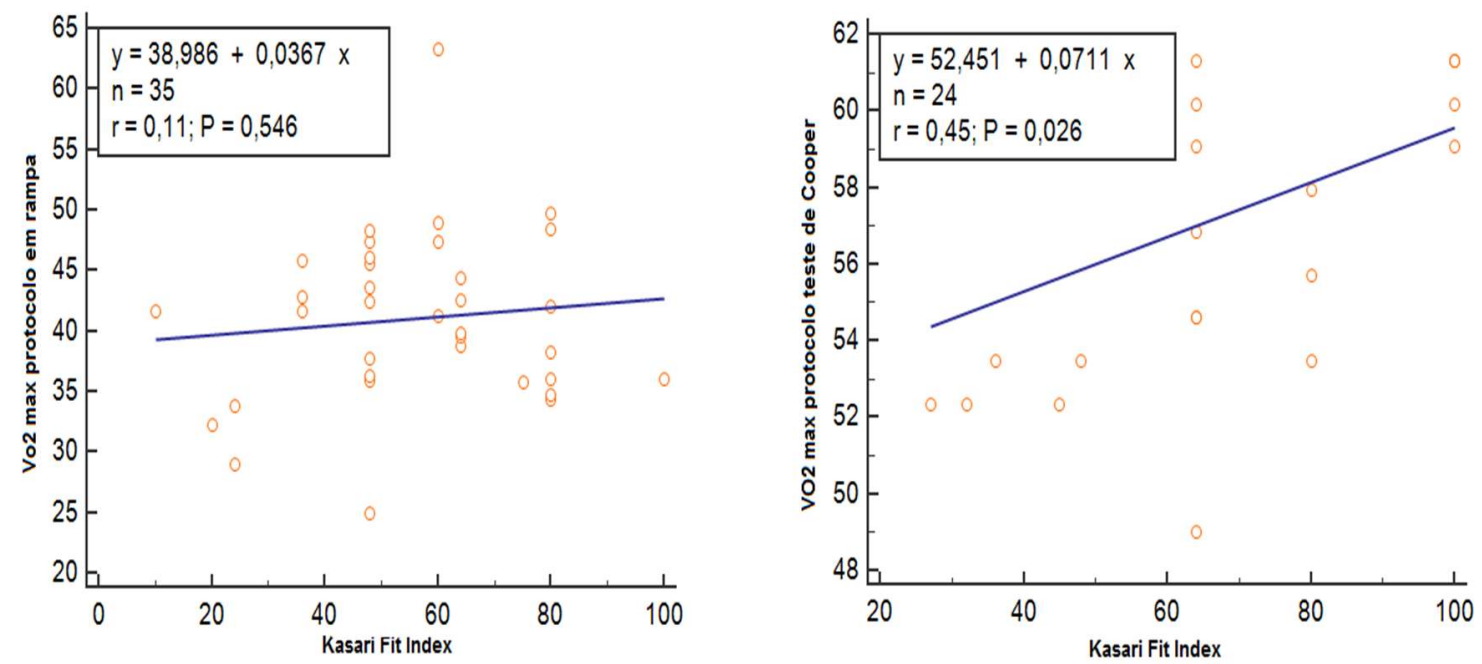

Figura 1 - Distribuição espacial não linear dos dados para melhor dimensão espacial 

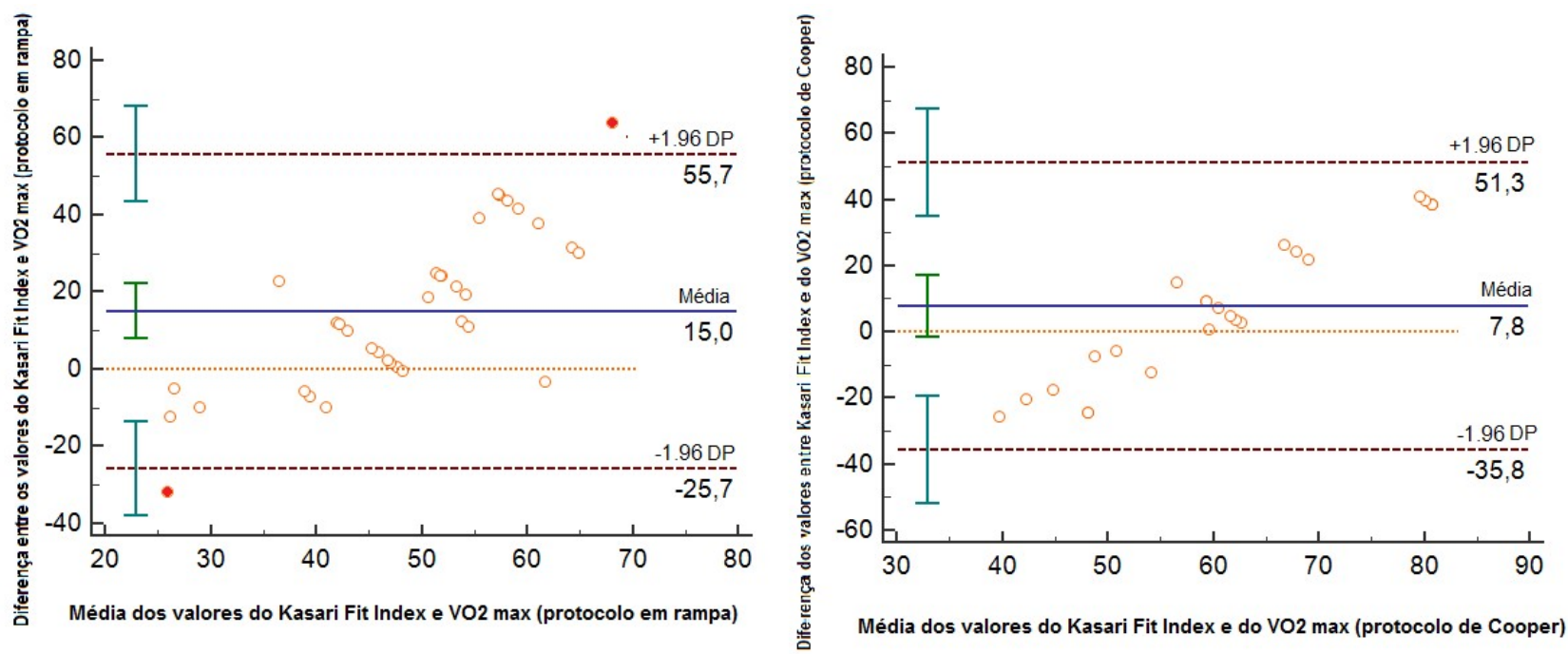

Figura 2 - Distribuição espacial não linear dos dados para melhor dimensão espacial

Tabela 3 - Valores plotados do Gráfico Bland-Altman

\begin{tabular}{|c|c|c|}
\hline & FIT $\times V^{2} O_{2 \text { maxral }}$ & FIT $x \mathrm{VO}_{2 \operatorname{maxCoo}}$ \\
\hline $\mathbf{N}$ & 35 & 24 \\
\hline M Diferenças & 14,99 & 7,77 \\
\hline $95 \%$ IC & $7,85 / 22,12$ & $-1,61 /-17,153$ \\
\hline$P\left(H_{0}: M=0\right)$ & 0,0001 & 0,10 \\
\hline Limite inferior & $-25,74$ & $-35,77$ \\
\hline 95\% IC Limite inferior & $-38,05 /-13,4$ & $-52,03 /-19,51$ \\
\hline Limite Superior & 55,71 & 51,32 \\
\hline 95\% IC Limite Superior & $43,39 / 68,02$ & $35,05 / 67,58$ \\
\hline
\end{tabular}

\section{Discussão}

O objetivo desta pesquisa foi verificar correlação e concordância entre o produto da intensidade, frequência e duração avaliados pelo Frequency-Intensity-Time Index of Kasari, o FIT, e o nível de capacidade cardiorrespiratória - através do $\mathrm{VO}_{2 \max }$ estimado - dos indivíduos avaliados, para verificar a pertinência da interpretação do FIT como estimativa de condicionamento físico.
Os resultados do presente estudo demonstraram que não houve correlação estatisticamente significante entre o FIT e o $\mathrm{VO}_{2 \text { maxrampa. Quando visualizam-se os }}$ resultados do gráfico Bland e Altmann, verifica-se uma média de diferença de 14,99 unidades entre os resultados, e uma variância entre os limites mínimo e máximo de $-25,74$ a 55,71 unidades. Além disso, a partir do BlandAltman, o resultado do teste t Student apontou 
que a concordância entre as medidas não se sustenta. Desta forma, identifica-se que há substancial sub e super estimação dos $\mathrm{VO}_{2 \text { maxrampa }}$ pelo FIT, o que torna estes testes não equivalentes. Para aqueles protocolos de avaliação da família militar que se apoiam na avaliação da capacidade cardiorrespiratória pelos testes de rampa, o FIT não deve ser usado como indicador do nível de condicionamento físico aeróbico.

Nos testes realizados entre o FIT e o

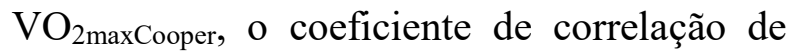
Person indicou uma correlação positiva e moderada. Porém, para o objetivo a que este estudo se propõe, este resultado é muito fraco para que possa ser considerado relevante. Avaliando ainda os resultados de Bland e Altman destes dados, a média das diferenças dos resultados apontou um valor de 7,8 unidades maior para o $\mathrm{VO}_{2 \text { maxCooper }}$ em relação ao FIT. Porém a variabilidade dos limites máximo e mínimo foi de 51 e - 35 unidades, respectivamente. Esta grande variabilidade dos resultados torna a medida imprecisa para estimação do condicionamento físico aeróbico.

Os resultados obtidos com os testes nos direcionam a inferir que a utilização do FIT não é uma alternativa eficaz para a avaliação do condicionamento físico dos militares e nem de seus familiares em situações que necessitem de informações auto reportadas.

Um dos possíveis motivos que levam a uma baixa correlação entre os dados, são os parâmetros subjetivos de avaliação de intensidade; os indivíduos, influenciados por fatores ambientais, idade, duração da atividade, entre outros, podem superestimar ou subestimar a intensidade(23). Desta forma, pode causar uma distorção considerável no resultado, visto que as variáveis intensidade, frequência e duração são multiplicadas entre si.

Explicam-se os resultados também pelo reconhecimento de que há a existência de uma variabilidade de efeitos da intensidade, frequência e duração do exercício sobre o $\mathrm{VO}_{2 \max }(24)$, ignorada pelo FrequencyIntensity-Time Index of Kasari, já que todas as três variáveis têm o mesmo peso para o FIT. Além disso, a duração das atividades físicas reportadas não é precisa, uma vez que, pessoas fisicamente ativas tendem a subestimar enquanto pessoas menos ativas são mais propensas a superestimar a duração; sendo que as atividades vigorosas tem a duração melhor reportada quando comparada às atividades moderadas e leves, por ser mais fácil de serem lembradas(25).

A idade exerce uma grande influência na medida da atividade física por meio de questionário; não só pelas diferenças em relação aos hábitos de atividades físicas, mas também pela precisão das informações fornecidas(26). Desta forma, podemos inferir que a maior dispersão das médias de idade dos indivíduos que participaram do protocolo rampa pode ter influência direta a ausência de correlação deste protocolo com o FIT. Já os alunos do Curso de Instrutores que tem uma dispersão de idade menor, obteve uma correlação moderada. A própria generalização do FIT, que não distingue idade ou sexo para a interpretação do condicionamento físico aeróbico pode ser questionada, uma vez que a influência destas variáveis nessa medida é bem conhecida(2).

Segundo Florindo e Hallal(27), não existe um questionário universal que seja aplicável a todos as pesquisas com atividades físicas pois para mensurá-la, é necessário um estudo minucioso de diversos fatores intervenientes no resultado. Apesar da existência de diversos questionários, correntes de estudo propõem a padronização da medida de atividade física por dados autorreportados, com intuito de gerar maior comparabilidade, porém sabe-se que é uma tarefa complexa devido aos fatores vinculados a atividade física $(28,29)$. Assim, a presente pesquisa, mesmo com suas limitações - amostra pequena e pouco variada, métodos indiretos de verificação do VO2máx contribui para o aumento do escopo de compreensão do fenômeno de preparo físico no Exército Brasileiro, seja da tropa ou da família militar.

O FIT pode ser tomado como uma medida de condicionamento físico ou como uma medida do nível de prática de exercício $(11,17)$, a partir do produto das respostas dadas em relação à intensidade, frequência e duração do exercício. Se tomarmos esta medida por esta última perspectiva, são aceitáveis correlações entre 0,30 e 0,50 do $\mathrm{VO}_{2 \max }$ com as medidas subjetivas do nível de atividade física(30), pois considera-se que uma boa parte da capacidade 
física é "constitucional" e não uma resposta ao treino e por que há uma interação complexa entre intensidade, frequência e duração do exercício com qualquer ganho de capacidade física(31). Nosso estudo gerou evidências nesse sentido, por isso, recomendamos estudos métricos futuros, tomando o FIT como uma medida de nível de atividade física (e não de condicionamento cardiorrespiratório), verificando evidências de validade de constructo, critério e confiabilidade (estabilidade temporal, repetibilidade e concordância interna).

\section{Pontos fortes e limitações do estudo}

O presente estudo apresenta como ponto forte a investigação da adequação do FIT como um instrumento de avaliação da aptidão cardiorrespiratória utilizando dois métodos distintos comparativos da avaliação do volume máximo de oxigênio, avaliado por medidas direta e indireta. Ademais, no que foi revelado pela revisão de literatura, foi o primeiro estudo com amostras de referências brasileiras conduzidas para verificar a adequação do método de medida em questão - o FIT - como estimador da aptidão cardiorrespiratória. Ainda, utilizou-se de amostra com poder de $95 \%$, evitando a ocorrência de erros tipo II nas inferências estatísticas realizadas.

Mas, há limitações a serem reconhecidas e reportadas. Por, a princípio, não haver diferença de sexo e idade nos níveis de aptidão cardiorrespiratória definidos pelo FIT, estas variáveis não foram controladas no presente estudo. Também não foi controlado o tipo de exercício físico praticado - se aeróbio, concorrente, resistido, por exemplo -algo que pode ser interessante para um estudo futuro.

\section{Conclusão}

Os dados geraram evidências de que não pode ser adequadamente suportado o uso do escore do FIT como uma medida de condicionamento físico aeróbico, especialmente quando considerada avaliação do $\mathrm{VO}_{2 \max }$ em protocolo de rampa, com amostra de grande dispersão de idade e padrões de prática de exercício. Por hora, como ainda não consta estudo sobre o uso do FIT como medida de nível de exercício físico, seria mais interessante usar o Frequency-Intensity-Time
Index of Kasari apenas como uma medida descritiva da prática de exercício, dando continuidade a essa linha de pesquisa com estudos métricos futuros.

\section{Declaração de conflito de interesses}

Não há nenhum conflito de interesses em relação ao presente estudo.

\section{Declaração de financiamento}

$\mathrm{O}$ presente estudo não recebeu financiamento de qualquer agência de fomento à pesquisa ou de qualquer outra fonte.

\section{Referências}

1. American College of Sports Medicine. ACSM's guidelines for exercise testing and prescription. $10^{\text {th }}$ ed. Philadelphia: Wolters Kluwer; 2018.

2. McArdle W, Katch F, Katch V. Exercise Physiology: Nutrition, Energy, and Human Performance. 8o ed. Philadelphia: Wolters Kluwer Health/Lippincott Williams \& Wilkins; 2015.

3. Herdy A, Caixeta A. Classificação Nacional da Aptidão Cardiorrespiratória pelo Consumo Máximo de Oxigênio. Arquivos Brasileiros de Cardiologia. 2016;106(5):389-95. http://dx.doi.org/10.5935/abc.20160070

4. De França E, Hirota VB, Caperuto E. Testes indiretos de VO2 máximo devem ser escolhidos de acordo com o gênero, variáveis antropométricas e capacidade aeróbica presumida. Revista Brasileira de Prescrição e Fisiologia do Exercício. 2014;8(49): 712-21.

5. Powers S, Howley E. Fisiologia do exercício - Teoria e aplicação ao condicionamento e ao desempenho. $9 \mathrm{o}$ ed. Barueri: Manole; 2017.

6. Brasil. Ministério da Defesa - Exército Brasileiro. Manual de Campanha EB20$M C$-10.350 - Treinamento Físico Militar. $4 \mathrm{o}$ ed. 2015.

7. Brasil. Ministério da Defesa - Exército Brasileiro. Portaria no 32 do Estado Maior do Exército, de 31 de março de 2008. 
8. Silva SC, Monteiro WD, Farinatti PTV. Avaliação da capacidade máxima de exercício: uma revisão sobre os protocolos tradicionais e a evolução dos modelos individualizados. Revista Brasileira de Medicina do Esporte. 2011;17(5):363369 . http://dx.doi.org/10.1590/S151786922011000500014

9. McDowell I. Measuring health: a guide to rating scales and questionnaires. Oxford University Press, USA; 2006.

10. Kasari D. The effects of exercise and fitness on serum lipids in college women. [Tese não publicada]. Mestrado em Fisiologia do fitness. Universidade de Montana, Missoula. 1976.

11. Sharkey BJ, Gaskill SE. Fitness \& health. Champaign, IL: Human Kinetics; 2013.

12. Godin G, Shephard RJ. A Simple Method to Assess Exercise Behavior in the Community. Canadian Journal of Applied Sport Science. 1985;10(3):1985;141-6.

13. Kriska AM, Knowler WC, LaPorte RE, Drash AL, Wing RR, Blair SN, et al. Development of Questionnaire to Examine Relationship of Physical Activity and Diabetes in Pima Indians. Diabetes Care. 1990;13(4):401-11. http://dx.doi.org/10.2337/diacare.13.4.40 1

14. Mauch JE, Birch JW. Guide to the successful thesis and dissertation: a handbook for students and faculty. 4o ed. New York: Marcel Ddkker; 1998.

15. Faul F, Erdfelder E, Lang A-G, Buchner A. G*Power 3: A flexible statistical power analysis program for the social, behavioral, and biomedical sciences. Behavior Research Methods. 2007;39(2):175-91.

http://dx.doi.org/10.3758/BF03193146

16. Balady GJ, Arena R, Sietsema K, Myers J, Coke L, Fletcher GF, et al. Clinician's Guide to Cardiopulmonary Exercise Testing in Adults: A Scientific Statement from the American Heart Association. Circulation. 2010;122(2):191-225. http://dx.doi.org/10.1161/CIR.0b013e318 $1 \mathrm{e} 52 \mathrm{e} 69$
17. Heyward V Stolarcyzk L. Applied body composition assessment. Champaign: Human Kinetics Books; 1996.

18. Callegari-Jacques S. Bioestatística: princípios e aplicações. Porto Alegre: Artmed; 2003.

19. Lin LI-K. A Concordance Correlation Coefficient to Evaluate Reproducibility. Biometrics. 1989;45(1):255.

20. Lin LI-K. Correction: a note on the concordance correlation coefficient. Biometrics. 2000;45(1):324-5.

21. McBride G. A proposal for strength-ofagreement criteria for Lin's Concordance Correlation Coefficient. Hamilton, New Zealand: National Institute of Water \& Atmospheric Research Ltd. 2005.

22. Bland JM, Altman DG. Statistical methods for assessing agreement between two methods of clinical measurement. The Lancet. 1986; 327(3476): 307-10. http://dx.doi.org/10.1016/S01406736(86)90837-8

23. Thelle DS. Assessment of physical activity and energy expenditure in epidemiological studies. European Journal of Epidemiology. 2007; 22(6): 351-2. http://dx.doi.org/10.1007/s10654007-9151-0

24. Wenger HA, Gordon JB. The interactions of intensity, frequency, and duration of exercise training in altering cardiorespiratory fitness. Sports Medicine. 1986; 3(5):346-56. https://dx.doi.org/10.2165/00007256198603050-00004

25. Sallis JF, Saelens BE. Assessment of Physical Activity by Self-Report: Status, Limitations, and Future Directions. Research Quarterly for Exercise and Sport. 2000;71(sup2):1-14. https://dx.doi.org/10.1080/02701367.200 0.11082780

26. Janz KF. Physical activity in epidemiology: moving from questionnaire to objective measurement. British Journal of Sports Medicine. 2006;40(3):191-2. 
http://dx.doi.org/10.1136/bjsm.2005.0230 36

27. Florindo AA, Hallal PC. Epidemiologia da Atividade Física. São Paulo: Atheneu; 2012. $210 \mathrm{p}$.

28. Shephard RJ. Limits to the measurement of habitual physical activity by questionnaires. British Journal of Sports Medicine. 2003;37(3):197-206. http://dx.doi.org/10.1136/bjsm.37.3.197

29. Pols MA, Peeters PHM, Kemper HCG, Grobbee DE. Methodological Aspects of Physical Activity Assessment in Epidemiological Studies. European Journal of Epidemiology. 1998;14(1):6370.

https://dx.doi.org/10.1023/A:1007427831 179

30. Farias Júnior JC de, Lopes A da S, Mota J, Santos MP, Ribeiro JC, Hallal PC. Validade e reprodutibilidade de um questionário para medida de atividade física em adolescentes: uma adaptação do Self-Administered Physical Activity Checklist. Revista Brasileira de Epidemiologia. 2012;15(1):198-210.

31. Shephard RJ. Assessment of physical activity and energy needs. American Journal of Clinical Nutrition. 1989;1195200.

http://dx.doi.org/10.1093/ajen/50.5.1195 